and $\chi_{x x}$ represent the coupling constants along the bond axis and normal to the bond axis, respectively. Under this transformation the $y$ axis corresponds to the $c$ axis and $\chi_{y y}=\chi_{c c}$. From the calculated value of the deviation from cylindrical symmetry $\eta$ in the bond axis system it is apparent that the charge distribution about the $\mathrm{S}-\mathrm{Cl}$ bond is approximately cylindrically symmetric. As pointed out by GoLDSTEIN ${ }^{21}$ the calculated value of $\eta$ should suggest about $4 \%$ double bond character.

Unfortunately no other microwave studies of a similar $\mathrm{S}-\mathrm{Cl}$ bond are to be found for comparison.
I thank Prof. Dr. H. D. Rudolph and Prof. Dr. H. DREIZLER for the invitation to their laboratory and for their interest in this work. I am further grateful to Dipl.-Phys. H. MäDER for writing a program and to the Deutsche Forschungsgemeinschaft and the Fonds der Chemie which supported this investigation. - Calculations were made with the IBM 7040 Computer of the Rechenzentrum der Universität Freiburg and with the Electrologica X 8 of the Rechenzentrum der Universität Kiel.

21 J. H. Goldstein, J. Chem. Phys. 24, 106 [1956].

\title{
Mikrowellenspektrum, Hinderungspotential der internen Rotation, Quadrupolkopplungskonstanten und Dipolmoment des 2-Methyl-Pyridins**
}

\author{
H. DreizleR *, H. D. Rudolph und H. Mäder * \\ Physikalisches Institut der Universität Freiburg \\ (Z. Naturforsch. 25 a, 25-35 [1970] ; eingegangen am 3. November 1969)
}

\begin{abstract}
The microwave rotational spectrum of 2-methyl-pyridine ( $\alpha$-picoline) has been investigated in the region from 6 to $30 \mathrm{kmc} / \mathrm{s}$. From the three lowest states of internal rotation $m=0,1,2$ the threeand sixfold components $V_{3}$ and $V_{6}$ of the potential barrier hindering the internal rotation have been determined to be $V_{3}=(258,4 \pm 0,1) \mathrm{cal} / \mathrm{mole}$ and $V_{6}=(-11,8 \pm 0,1) \mathrm{cal} / \mathrm{mole}$. From the splitting of low- $J$ lines $m=0$ the nuclear quadrupole coupling constants for the ${ }^{14} \mathrm{~N}$ nucleus have been derived as $\chi_{a a}=(-0,33 \pm 0,02) \mathrm{mc} / \mathrm{s}, \chi_{b b}=(-2,86 \pm 0,02) \mathrm{mc} / \mathrm{s}, \chi_{c c}=(+3,19 \pm 0,02) \mathrm{mc} / \mathrm{s}$. The hyperfine structure of rotational transitions in excited states of internal rotation could also be accounted for with these coupling constants. The dipole moment components derived from Stark-effect measurements in the ground torsional state $m=0$ are $\mu_{a}=(0,72 \pm 0,01)$ Debye and $\mu_{b}=(1,71 \pm 0,02)$ Debye.
\end{abstract}

Das Rotationsspektrum des 2-Methyl-Pyridins (Abb. 1) - im folgenden 2MP genannt - wurde untersucht, um das Hinderungspotential der internen Rotation des symmetrischen $\mathrm{CH}_{3}$-Kreisels bezüglich des asymmetrischen Rumpfes (Pyridinring) zu bestimmen. Den Rechnungen liegt ein Molekülmodell zugrunde, das sich aus einem ebenen, starr angenommenen Pyridinring (frame) und einer dagegen behindert drehbaren starren symmetrischen Methylgruppe (top) zusammensetzt.

Wegen der $\mathrm{C}_{3 \mathrm{v}}$-Symmetrie des Teilkreisels bezüglich der Achse der internen Rotation besitzt das Hinderungspotential drei äquivalente Minima und kann in eine Cosinusreihe entwickelt werden, die mit einem dreizähligen Term beginnt:

$V(\alpha)=\frac{1}{2} V_{3}(1-\cos 3 \alpha)+\frac{1}{2} V_{6}(1-\cos 6 \alpha)+\ldots$

* Neue Anschrift: Institut für Physikalische Chemie, Abt. Chemische Physik, 23 Kiel, Ohlshausenstraße 40-60.
Das Hinderungspotential ist auf Grund der Spiegelsymmetrie des Molekülrumpfes eine gerade Funktion in dem Winkel $\alpha$, der die relative Drehlage des Teilkreisels gegenüber dem Rumpf angibt.

Wegen der Kopplung von Gesamtrotation und interner Rotation fallen die Rotationsspektren verschieden angeregter interner Rotationszustände im allgemeinen nicht zusammen und erlauben somit Rückschlüsse auf die verschiedenzähligen Potentialterme in der Entwicklung (1). In der vorliegenden Arbeit gelang es, die beiden ersten Potentialkoeffizienten $V_{3}$ und $V_{6} \mathrm{zu}$ bestimmen. Über höhere Potentialterme konnte keine Aussage gemacht werden, da die gemessenen Rotationsübergänge von ihnen viel weniger abhängen als von den beiden ersten Potentialkoeffizienten. Zur Bestimmung von $V_{3}$ und $V_{6}$ mußten allerdings neben dem Rotationsspektrum im

** Teil der Diplomarbeit H. MädER, Freiburg 1968. 


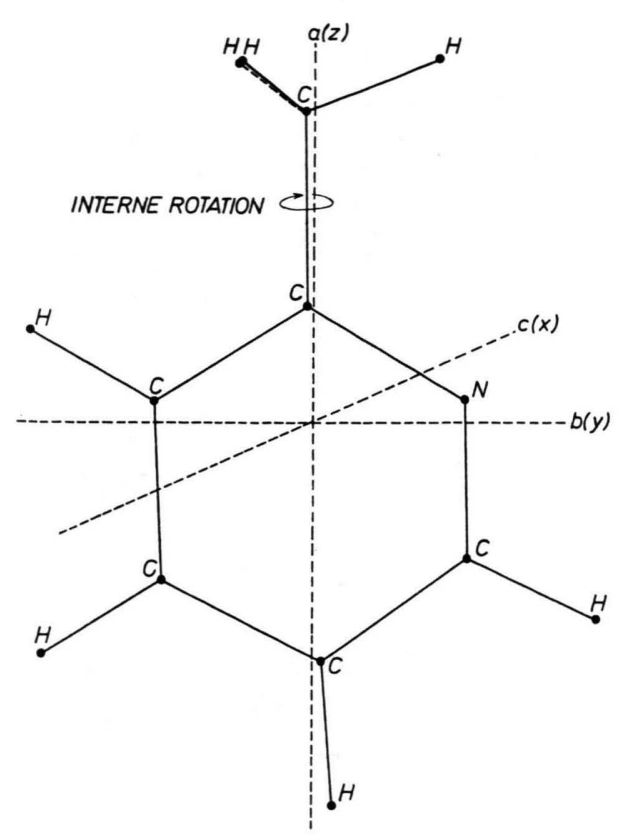

Abb. 1. $2 \mathrm{MP}$, Gestalt und Hauptachsenlage. Die $\mathrm{C}-\mathrm{CH}_{3}$ Achse liegt in der Ringebene ( $a, b$-Ebene) und fällt fast mit der $a$-Achse zusammen.

Grundzustand der internen Rotation auch die Rotationsspektren angeregter interner Rotationszustände zugeordnet werden, da aus einem Spektrum allein nicht mehr als ein Potentialterm bestimmbar ist.

\section{Experimentelles}

Das Mikrowellenspektrum von 2MP wurde zwischen 6 und $30 \mathrm{GHz}$ untersucht und partiell zugeordnet. Dabei wurden 32 - und $100 \mathrm{kHz}$-modulierte Stark-Effekt-Spektrographen verwendet, die bereits an anderer Stelle ${ }^{\mathbf{1}^{-3}}$ ausführlich beschrieben sind.

Der Spektrograph mit $32 \mathrm{kHz}$-Stark-Effektmodulation wurde zur Ausmessung der Hyperfeinstruktur der Linien verwendet. Er zeichnet sich mit einem Auflösungsvermögen von ca. $100 \mathrm{kHz}$ - allerdings bei kleinerem Signal-Rauschverhältnis - gegenüber dem 100 kHz-Stark-Effektmodulierten Spektrographen (Auflösungsvermögen ca. $300 \mathrm{kHz}$ ) aus. Die Substanz ${ }^{4}$ war käuflich erhältlich. Sie wurde in das

1 H. D. Rudolph, Z. Angew. Phys. 13, 401 [1961].

2 H. D. Rudolph u. H. Seiler, Z. Naturforsch. 20 a, 1682 [1965].

3 H. D. Rudolph, H. Dreizler, A. Jaeschke u. W. WendLING, Z. Naturforsch. 22 a, 940 [1967].

4 2-Methyl-Pyridin purum (a-Picolin), Fa. Merck, Darmstadt.
Probengefäß des Spektrographen eindestilliert, sonst aber nicht weiter gereinigt. Bei den Messungen wurde die Absorptionszelle auf ca. $-30{ }^{\circ} \mathrm{C}$ bis $-50{ }^{\circ} \mathrm{C}$ gekühlt. Bei diesen Temperaturen hat die Substanz einen geeigneten Dampfdruck von ca. 20 bis $5 \mathrm{mT}$.

\section{Theorie}

Das eingangs beschriebene Molekülmodell, dem die Analyse des Spektrum zugrunde liegt, ist bereits mehrfach ${ }^{5-7}$ beschrieben worden. Eine ausführliche Diskussion des Spezialfalles, in dem die interne Rotationsachse in der Rumpfebene ( $a, b$-Ebene) liegt, findet sich bei Rudolph und Trinkaus ${ }^{8}$.

Der Hamilton-Operator, dessen Invarianzgruppe $\mathrm{zu} \mathrm{D}_{3}$ isomorph ist, hat dann die Form

$$
\begin{aligned}
H= & \frac{1}{2}\left(C+B+F \lambda_{b}^{2}\left(\frac{I_{a}}{I_{b}}\right)^{2}\right)\left(P_{c}^{2}+P_{b}^{2}\right) \\
& +\left(A+F \lambda_{a}^{2}\left(\frac{I_{a}}{I_{a}}\right)^{2}\right) P_{a}^{2} \\
& +\frac{1}{2}\left(C-B-F \lambda_{b}^{2}\left(\frac{I_{a}}{I_{b}}\right)^{2}\right)\left(P_{c}^{2}-P_{b}^{2}\right) \\
& +F p^{2} \\
& +\frac{1}{2} V_{3}(1-\cos 3 \alpha)+\frac{1}{2} V_{6}(1-\cos 6 \alpha) \\
& +F \lambda_{a} \lambda_{b}\left(\frac{I_{a}}{I_{a}}\right)\left(\frac{I_{a}}{I_{b}}\right)\left(P_{a} P_{b}+P_{b} P_{a}\right) \\
& -2 F p\left(\lambda_{a} \frac{I_{a}}{I_{a}} P_{a}+\lambda_{b} \frac{I_{a}}{I_{b}} P_{b}\right) .
\end{aligned}
$$

Hierbei bedeuten:

$A, B, C$ Rotationskonstanten des Gesamtmoleküls (interne Rotation eingefroren) $A=h /\left(8 \pi^{2} I_{a}\right)$ etc., $I_{g}: g$-Hauptträgheitsmomente $(g=a, b, c)$;

$P_{g} \quad$ Operator der Komponente des Gesamtdrehimpulses in Richtung der Hauptachse $g$;

$\lambda_{g}$ Richtungskosinus der $\mathrm{CH}_{3}$-Achse relativ zur $g$-Achse;

$I_{\alpha}$ Trägheitsmoment der $\mathrm{CH}_{3}$-Gruppe um ihre Symmetrieachse;

$F=h /\left[8 \pi^{2} I_{\alpha}\left(1-\lambda_{a}^{2}\left(I_{\alpha} / I_{a}\right)^{2}-\lambda_{b}\left(I_{\alpha} / I_{b}\right)^{2}\right)\right]$, „reduzierte" Rotationskonstante für die interne Rotation des Moleküls;

5 D. R. Herschbach, J. Chem. Phys. 31, 91 [1959].

6 C. C. Lin u. J. D. Swalen, Rev. Mod. Phys. 31, 841 [1959].

7 H. Dreizler, Fortschr. Chem. Forsch. 10, 59 [1968].

8 H. D. Rudolph u. A. Trinkaus, Z. Naturforsch. 23 a, 68 [1968]. 
$p=-i \frac{\partial}{\partial \alpha}$ Operator der Komponente des Gesamtdrehimpulses der $\mathrm{CH}_{3}$-Gruppe in Richtung ihrer Symmetrieachse.

Für die numerische Ermittlung der Energieeigenwerte von $H$ erwies es sich für das $2 \mathrm{MP}$ als zweckmäßig, vom Grenzfall der freien internen Rotation auszugehen. Ein geeignetes Basissystem von Funktionen, die allerdings noch nicht bezüglich $\mathrm{D}_{3}$ symmetrisiert sind, ist dann das Produkt der Eigenfunktionen von (2.1), den von den drei Euler-Winkeln abhängigen symmetrischen Kreiselfunktionen, und von (2.3), den normierten Eigenfunktionen eines starren Rotators mit fester Achse, das sind die Funktionen:

$|J K M m\rangle=\psi_{J K M}^{\times} \cdot \frac{1}{\sqrt{2 \pi}} e^{i m \alpha}\left(\psi_{J K M}^{\times} \quad\right.$ für $\left.\quad m=0\right)$

Die Energiematrix ist diagonal in $J$ und $M$ und jede der in $m$ unendlichen $J M$-Submatrizen kann in einzelne Submatrizen mit jeweils festem $m$ angeordnet werden, die aber auf Grund des Potentials (2.4) durch Matrixelemente verbunden sind. Die einzelnen $m$-Blöcke sind nichtdiagonal in $K$ auf Grund der Asymmetrie des Moleküls (2.2), sowie der Wechselwirkungsoperatoren (2.5) und (2.6) von interner Rotation und Gesamtrotation. Wie aus der Gestalt der Energiematrix ${ }^{7,8}$ ersichtlich ist, tritt eine Faktorisierung in drei Untermatrizen auf, die durch die Sätze

$m=3 n-1, \quad m=3 n, m=3 n+1 \quad(n=0, \pm 1, \ldots)$

gekennzeichnet sind. Der erste und letzte Submatrizensatz läßt sich durch eine orthogonale Transformation ineinander überführen und erbringt also identische Eigenwerte, die der zweidimensionalen Darstellung $\mathrm{E}$ von $\mathrm{D}_{3}$ zuzuordnen sind. Der zu $m=3 n$ gehörige Submatrizensatz führt bei nichtverschwindendem Potential zu einfachen Eigenwerten und kann durch eine spezielle Transformation ${ }^{7,8}$ nach den Spezies $A_{1}, A_{2}$ von $D_{3}$ faktorisiert werden. Funktionsbasen für $A_{1}$ und $A_{2}$ sind dann die Funktionen

$$
\begin{gathered}
|J| K|\gamma M| m|\delta\rangle=\psi_{J|K| \gamma M|m| \gamma} \\
=\frac{1}{2 \sqrt{\pi}} S^{J|K| \gamma M}\left(e^{i|m| \alpha}+(-1)^{K+\gamma+\delta} e^{-i|m| a}\right) \\
\delta=0,1 \quad \text { für }|m|=3 n, \quad n=1,2, \ldots \\
|J| K|\gamma M 00\rangle=S^{J|K| \gamma M} \quad \text { für } \quad m=0
\end{gathered}
$$

mit den Wang-Funktionen

$$
\begin{aligned}
& S_{J|K| \gamma M}=\frac{1}{\sqrt{2}}\left((-1)^{\gamma} \psi_{J-K M}^{\times}+\psi_{J K M}^{\times}\right) \\
& \gamma=0,1 \text { für } K \neq 0 \\
& S_{J 00 M}=\psi_{J 0 M}^{\times} \\
& \text {für } K=0 .
\end{aligned}
$$

Die Spezieszugehörigkeit der Energieeigenwerte zu $A_{1}, A_{2}$ läßt sich durch den Index $\delta=0,1$ für $|m|=3 n, n=1,2, \ldots$ bzw. $\gamma=0,1$ für $m=0$ kennzeichnen ${ }^{8}$.

Zur Ermittlung der Energieeigenwerte für $m \neq 0$ wurde die Energiematrix (2) in den bezüglich $D_{3}$ symmetrisierten Funktionsbasen aufgestellt und unter Einbeziehung eines genügend großen Teilausschnitts aus der in $m$ unendlichen $J M$-Submatrix diagonalisiert. Für das 2MP mußten die drei dem betreffenden $m$-Block nächstbenachbarten durch das Hinderungspotential verbundenen $m$-Blöcke, also insgesamt 7 für die E-Spezies, berücksichtigt werden, um die Frequenzen innerhalb der Meßgenauigkeit zu berechnen. Für das Rotationsspektrum im Grundzustand der internen Rotation $m=0$ wurde der Energieoperator eines korrigierten quasistarren asymmetrischen Kreisels zugrunde gelegt (vgl. folgenden Abschnitt).

\section{Spektrum und Hinderungspotential}

Die Rotationsspektren lassen sich nach der Quantenzahl $m$ klassifizieren, die nur für verschwindendes Hinderungspotential eine gute Quantenzahl darstellt. Die Spektren lassen sich nun in zwei Klassen einteilen: dem Rotationsspektrum im Grundzustand der internen Rotation $m=0$, aus dem die Kernquadrupolkopplungskonstanten und Dipolmomentkomponenten des 2MP bestimmt wurden, und den Rotationsspektren in angeregten internen Rotationszuständen $m \neq 0$, die zusammen mit dem $m=0$-Spektrum die im Hamilton-Operator (2) auftretenden spektroskopischen Konstanten, insbesondere die Potentialkoeffizienten $V_{3}$ und $V_{6}$, erbrachten.

$$
m=0
$$

Das Rotationsspektrum im Grundzustand der internen Rotation läßt sich im allgemeinen gut an das Spektrum nach dem Modell eines starren asymmetrischen Kreisels anpassen. Für verschwindendes Potential ist dies im Rahmen des verwendeten Modells exakt: der Diagonalblock $m=0$ ist nämlich formal die Energiematrix eines starren asymmetrischen Kreisels mit auf Grund der Wechselwirkung von interner und äußerer Rotation modifizierten Rotations- 
konstanten [bis auf den Kreuzterm (2.5), der sich durch eine Drehtransformation in der $a, b$-Ebene beseitigen läßt]. Entsprechend ergibt sich auch im Grenzfall unendlich hohen Hinderungspotentials (interne Rotation eingefroren) ein starres Kreiselspektrum; die Rotationskonstanten sind dann die des Gesamtmoleküls. Auch für endlich hohe Potentiale hat sich bislang die Beschreibung des $m=0$-Spektrums (bzw. $v=0, \sigma=0$ im Grenzfall hohen Hinderungspotentials ${ }^{5-7}$ ) durch das Spektrum eines effektiven starren Kreisels bewährt. Im Falle des 2MP erwies sich allerdings eine solche Behandlung als unzulänglich. Bei der Anpassung von Rotationsübergängen von $J=0$ bis $J=4$ an das Modell eines starren asymmetrischen Kreisels zeigte es sich, daß die Differenz von gemessenen und berechneten Frequenzen für einzelne Linien bis zu $2 \mathrm{MHz}$ betrugen. Diese Abweichungen überschreiten die Größe der Zentrifugalaufweitung vergleichbarer Moleküle $(<100 \mathrm{kHz})$ und sind der Wechselwirkung von interner und äußerer Rotation zuzuschreiben. Eine Anpassung nach dem Gesamt-Hamilton-Operator (2) ist nicht möglich, da die dort auftretenden Konstanten a priori nicht bekannt sind und sich aus dem $m=0$-Spektrum allein nicht bestimmen lassen. Es wurde deshalb versucht, ausgehend von dem effektiven Hamilton-Operator, der im allgemeinen zur Ermittlung der Energieeigenwerte im Grenzfall hohen Hinderungspotentials angewandt wird ${ }^{5-7}$

$$
\begin{aligned}
H_{v=0, \sigma=0} & =A P_{a}^{2}+B P_{b}^{2}+C P_{c}^{2} \\
& +F \sum_{n} W_{00}^{(2 n)}\left(\lambda_{a}\left(I_{\alpha} / I_{a}\right) P_{a}+\lambda_{b}\left(I_{\alpha} / I_{b}\right) P_{b}\right)^{2 n}
\end{aligned}
$$

die Anpassung zu verbessern. Überschlägige Strukturrechnungen ergaben für den Winkel zwischen interner Rotationsachse und $a$-Achse einen Wert kleiner als $2^{\circ}$, so daß in erster Näherung $\lambda_{b}$ vernachlässigbar ist und $\lambda_{a}=1$ gesetzt werden kann. Zieht man ferner den Term $F W_{00}^{(2)}\left(I_{\alpha} / I_{a}\right)^{2} P_{a}^{2}$ in den Rotationsanteil und vernachlässigt Glieder höherer als 4. Ordnung in (5.1), so erhält man den Energieoperator eines korrigierten quasistarren Kreisels ${ }^{9}$ :

mit

$$
H=A^{\prime} P_{a}^{2}+B^{\prime} P_{b}^{2}+C^{\prime} P_{c}^{2}+\tau P_{a}^{4}
$$

$$
A^{\prime}=A+F W_{00}^{(2)}\left(I_{\alpha} / I_{a}\right)^{2}, \quad \tau=F W_{00}^{(4)}\left(I_{\alpha} / I_{a}\right)^{4}
$$

(der konstante Term $F W_{00}^{(0)}$ ist weggelassen).

9 Wegen der obigen Vernachlässigung werden auch die Rotationskonstanten $B$ und $C$ des Gesamtmoleküls bei der Anpassung nach (5.2) geringfügig modifiziert und deshalb gestrichen.
Berücksichtigt man den Korrekturterm $\tau P_{a}{ }^{4}$ in erster Ordnung, so ergibt sich für die Energiewerte $E_{J K_{-} K_{+}}=\frac{1}{2}\left(A^{\prime}+C^{\prime}\right) J(J+1)$ $+\frac{1}{2}\left(A^{\prime}-C^{\prime}\right) E_{J K_{-} K_{+}}(\varkappa)+\tau\left\langle P_{a}^{4}\right\rangle$

$\left\langle P_{a}{ }^{4}\right\rangle \quad$ Erwartungswert von $P_{a}^{4}$ im Zustand $J K_{-} K_{+}$;

$\varkappa=\left(2 B^{\prime}-A^{\prime}-C^{\prime}\right) /\left(A^{\prime}-C^{\prime}\right)$ Asymmetrieparameter; $E(\varkappa) \quad$ "reduzierte“ Energie eines starren Kreisels; $K_{-} K_{+}$Pseudoquantenzahl zur Numerierung der Eigenwerte des starren asymmetrischen Kreisels.

Die Niveaufolge und Auswahlregeln des starren asymmetrischen Kreisels bleiben erhalten, da die Korrektur klein ist. Das Dipolmoment des 2MP liegt in der Rumpfebene; es wurde ein gegenüber dem $a$-Spektrum rund sechsmal intensiveres $b$-Spektrum gefunden. Die Werte von $\left\langle P_{a}{ }^{4}\right\rangle$ sind grob genähert durch $K_{-}^{4}$ gegeben ${ }^{10}$, wodurch sich die besonders großen Abweichungen des $b$-Spektrums $\left(\Delta K_{-}= \pm 1\right)$ vom Spektrum eines starren asymmetrischen Kreisels bei Übergängen zwischen Niveau mit hohem $K_{\text {_ }}$ erklären (maximale Abweichung beim Übergang $\left.4_{40}-4_{31}: 2,12 \mathrm{MHz}\right)$.

Eine Anpassung der Linien $m=0$ bis $J=4$ nach (5.3) erbrachte eine um etwa den Faktor 20 kleinere Standardabweichung von $26 \mathrm{kHz}$ als bei der Anpassung nach dem Modell des starren asymmetrischen Kreisels.

Aus der Anpassung des Korrekturkoeffizienten $\tau$ ergibt sich die Möglichkeit, die Höhe des Hinderungspotentials abzuschätzen. Die zu $\tau$ proportionalen Störkoeffizienten $W_{00}^{(4)}$ liegen nämlich in Abhängig. keit von der Potentialhöhe tabelliert vor ${ }^{5}$. Es läßt sich allerdings nur der Potentialkoeffizient $V_{3}$ bestimmen, da die Zuordnung eines Störkoeffizienten $W_{00}^{(4)}$ zu einem Satz von mehr als einem Potentialterm nicht eindeutig ist. Setzt $\operatorname{man} V_{6}=V_{9}=\ldots=0$, so erhält man für $V_{3}$ einen effektiven Wert, in den höhere Potentialkoeffizienten summarisch eingehen, und zwar $V_{3}=250 \mathrm{kcal} / \mathrm{Mol}$. Eine mit dieser Methode konsistente Möglichkeit zur Abschätzung von $V_{3}$ liegt wegen der Planarität des Rumpfes in der Berechnung des Trägheitsdefektes ${ }^{12}$ von $2 \mathrm{MP}$, der wegen der auf Grund der Wechselwirkung von in-

10 Bei Zuordnung der Hauptachse $a$ zur Quantisierungsachse $z$ (Darstellung $I^{\mathrm{r}}$ oder $I^{\mathrm{l}}$ ).

12 H. D. Rudolph, noch nicht veröffentlicht, auszugsweise vorgetragen auf dem Congress on High Resolution Spectroscopy, Dijon 1969. 


\begin{tabular}{|c|c|c|c|c|c|c|c|c|}
\hline $\begin{array}{c}\text { Übergang } \\
J_{K_{-} K_{+}-J^{\prime}{ }^{\prime}{ }_{-} K_{+}^{\prime}}\end{array}$ & $F-F^{\prime}$ & $\begin{array}{c}\nu_{\exp } \\
\mathrm{MHz}\end{array}$ & $\begin{array}{c}\bar{v}_{\exp } \\
\text { oder } v_{\exp } \\
\mathrm{MHz}\end{array}$ & $\begin{array}{c}\nu_{\mathrm{ber}} \\
\mathrm{MHz}\end{array}$ & $\begin{array}{l}\Delta v_{\exp } \\
\mathrm{MHz}\end{array}$ & $\begin{array}{l}\Delta \nu_{\text {ber }} \\
\mathrm{MHz}\end{array}$ & $\bar{v}_{\exp }-v_{\mathrm{ber}}$ & $\begin{array}{l}\text { Bemer- } \\
\text { kungen }\end{array}$ \\
\hline $0_{00}-1_{11}$ & $\begin{array}{l}2-1 \\
1-1 \\
0-1\end{array}$ & $\begin{array}{l}7587,52 \\
7586,68 \\
7588,80\end{array}$ & 7587,38 & 7587,383 & $\begin{array}{r}0,14 \\
-0,70 \\
1,42\end{array}$ & $\begin{array}{r}0,14 \\
-0,71 \\
1,43\end{array}$ & -3 & $\mathrm{ec}, \mathrm{hfs}$ \\
\hline $1_{01}-2_{02}$ & & & 8768,29 & 8768,305 & & & -15 & \\
\hline $1_{10}-2_{11}$ & & & 9718,37 & 9718,363 & & & +7 & \\
\hline $1_{11}-2_{12}$ & & & 8094,75 & 8094,790 & & & -40 & \\
\hline $1_{01}-2_{12}$ & & & 11228,93 & 11228,884 & & & +46 & $\mathrm{ec}$ \\
\hline $1_{10}-2_{21}$ & $\begin{array}{l}3-2 \\
2-1 \\
1-0\end{array}$ & $\begin{array}{l}19120,49 \\
19119,60 \\
19121,90\end{array}$ & 19120,35 & 19120,358 & $\begin{array}{r}0,14 \\
-0,75 \\
1,55\end{array}$ & $\begin{array}{r}0,14 \\
-0,72 \\
1,51\end{array}$ & -8 & $\mathrm{ec}$ \\
\hline $1_{11}-2_{02}$ & $\begin{array}{l}3-2 \\
2-1\end{array}$ & $\begin{array}{l}5634,10 \\
5634,64\end{array}$ & 5634,17 & 5634,210 & $\begin{array}{r}-0,07 \\
0,47\end{array}$ & $\begin{array}{r}-0,07 \\
0,46\end{array}$ & -40 & $\mathrm{ec}$ \\
\hline $1_{11}-2_{20}$ & $\begin{array}{l}3-2 \\
2-1 \\
1-0 \\
2-2 \\
1-1\end{array}$ & $\begin{array}{l}20070,24 \\
20071,43 \\
20068,76 \\
20070,55 \\
20070,90\end{array}$ & 20070,45 & 20070,416 & $\begin{array}{r}-0,21 \\
0,98 \\
-1,69 \\
0,10 \\
0,45\end{array}$ & $\begin{array}{r}-0,21 \\
0,97 \\
-1,68 \\
0,11 \\
0,46\end{array}$ & +34 & ec, hfs \\
\hline $2_{20}-2_{11}$ & $\begin{array}{l}3-3 \\
2-2 \\
1-1\end{array}$ & $\begin{array}{l}9540,39 \\
9539,80 \\
9540,70\end{array}$ & 9540,25 & 9540,266 & $\begin{array}{r}0,14 \\
-0,45 \\
0,45\end{array}$ & $\begin{array}{r}0,13 \\
-0,46 \\
0,46\end{array}$ & -16 & $\mathrm{ec}, \mathrm{hfs}$ \\
\hline $2_{21}-2_{12}$ & $\begin{array}{l}3-3 \\
2-2 \\
1-1\end{array}$ & $\begin{array}{l}11837,10 \\
11838,21 \\
11836,46\end{array}$ & 11837,35 & 11837,355 & $\begin{array}{r}-0,25 \\
0,86 \\
-0,89\end{array}$ & $\begin{array}{r}-0,25 \\
0,88 \\
-0,88\end{array}$ & -5 & $\mathrm{ec}, \mathrm{hfs}$ \\
\hline $22_{20}-3_{21}$ & & & 13888,33 & 13888,308 & & & +22 & ec \\
\hline $2_{02}-3_{03}$ & $\begin{array}{l}4-3 \\
3-2\end{array}$ & $\begin{array}{l}12831,52 \\
12831,19\end{array}$ & 12831,42 & 12831,421 & $\begin{array}{r}0,10 \\
-0,23\end{array}$ & $\begin{array}{r}0,09 \\
-0,22\end{array}$ & -1 & $\mathrm{ec}$ \\
\hline $2_{11}-3_{12}$ & $\begin{array}{l}4-3 \\
2-1\end{array}$ & $\begin{array}{l}14482,40 \\
14482,64\end{array}$ & 14482,42 & 14482,417 & $\begin{array}{r}-0,02 \\
0,22\end{array}$ & $\begin{array}{r}-0,01 \\
0,21\end{array}$ & +3 & ec \\
\hline $2_{21}-3_{22}$ & & & 13359,83 & 13359,865 & & & -35 & ec \\
\hline $2_{02}-3_{13}$ & $\begin{array}{l}4-3 \\
3-2 \\
2-1\end{array}$ & $\begin{array}{l}14522,85 \\
14522,03 \\
14523,07\end{array}$ & 14522,63 & 14522,616 & $\begin{array}{r}0,22 \\
-0,60 \\
0,44\end{array}$ & $\begin{array}{r}0,22 \\
-0,61 \\
0,44\end{array}$ & +14 & ec, hfs \\
\hline $2_{12}-3_{03}$ & $\begin{array}{l}4-3 \\
3-2 \\
2-1\end{array}$ & $\begin{array}{l}10370,79 \\
10371,16 \\
10370,43\end{array}$ & 10370,85 & 10370,842 & $\begin{array}{r}-0,06 \\
0,31 \\
-0,42\end{array}$ & $\begin{array}{r}-0,07 \\
0,32 \\
-0,42\end{array}$ & +8 & ec, hfs \\
\hline $3_{30}-3_{21}$ & $\begin{array}{l}4-4 \\
3-3\end{array}$ & $\begin{array}{l}17126,99 \\
17126,67\end{array}$ & 17126,91 & 17126,906 & $\begin{array}{r}0,08 \\
-0,24\end{array}$ & $\begin{array}{r}0,08 \\
-0,23\end{array}$ & +4 & $\mathrm{ec}$ \\
\hline $3_{21}-3_{12}$ & $\begin{array}{l}4-4 \\
3-3\end{array}$ & $\begin{array}{l}8946,24 \\
8945,90\end{array}$ & 8946,16 & 8946,158 & $\begin{array}{r}0,08 \\
-0,26\end{array}$ & $\begin{array}{r}0,08 \\
-0,24\end{array}$ & +2 & $\mathrm{ec}, \mathrm{st}$ \\
\hline $3_{12}-3_{03}$ & $\begin{array}{l}4-4 \\
3-3 \\
2-2\end{array}$ & $\begin{array}{l}6546,57 \\
6548,04 \\
6546,08\end{array}$ & 6546,94 & 6546,935 & $\begin{array}{r}-0,37 \\
1,10 \\
-0,86\end{array}$ & $\begin{array}{r}-0,37 \\
1,11 \\
-0,89\end{array}$ & +5 & $\mathrm{ec}, \mathrm{hfs}$ \\
\hline $3_{31}-3_{22}$ & $\begin{array}{l}4-4 \\
3-3\end{array}$ & $\begin{array}{l}17778,57 \\
17778,78\end{array}$ & 17778,63 & 17778,640 & $\begin{array}{r}-0,06 \\
0,15\end{array}$ & $\begin{array}{r}-0,05 \\
0,15\end{array}$ & -10 & $\mathrm{ec}$ \\
\hline $3_{22}-3_{13}$ & $\begin{array}{l}4-4 \\
3-3\end{array}$ & $\begin{array}{l}13134,91 \\
13136,01\end{array}$ & 13135,20 & 13135,183 & $\begin{array}{r}0,29 \\
0,86\end{array}$ & $\begin{array}{r}0,29 \\
0,86\end{array}$ & +17 & ec \\
\hline $3_{21}-4_{22}$ & $\begin{array}{l}5-4 \\
4-3\end{array}$ & $\begin{array}{l}18896,98 \\
18897,20\end{array}$ & 18897,05 & $18,897,047$ & $\begin{array}{r}-0,07 \\
0,15\end{array}$ & $\begin{array}{r}-0,07 \\
0,15\end{array}$ & +3 & $\mathrm{ec}$ \\
\hline $3_{03}-4_{04}$ & $\begin{array}{l}5-4 \\
4-3\end{array}$ & $\begin{array}{l}16619,69 \\
16619,37\end{array}$ & 16619,59 & 16619,586 & $\begin{array}{r}0,10 \\
-0,22\end{array}$ & $\begin{array}{r}0,10 \\
-0,22\end{array}$ & +4 & ec \\
\hline $3_{30}-4_{31}$ & & & 18138,61 & 18138,580 & & & +30 & $\mathrm{ec}$ \\
\hline $3_{12}-4_{13}$ & & & 19111,25 & 19111,300 & & & -50 & $\mathrm{ec}$ \\
\hline $3_{31}-4_{32}$ & & & 18050,46 & 18050,465 & & & -5 & $\mathrm{ec}$ \\
\hline $3_{13}-4_{14}$ & & & 15952,25 & 15952,267 & & & -17 & $\mathrm{ec}$ \\
\hline $3_{22}-4_{23}$ & & & 17704,76 & 17704,823 & & & -63 & $\mathrm{ec}$ \\
\hline
\end{tabular}

Tab. 1 . 


\begin{tabular}{|c|c|c|c|c|c|c|c|c|}
\hline $\begin{array}{l}\text { Utbergang } \\
J_{K_{-} K_{+}-} J_{K_{-}^{\prime} K_{+}^{\prime}}\end{array}$ & $F-F^{\prime}$ & $\begin{array}{l}v_{\exp } \\
\mathrm{MHz}\end{array}$ & $\begin{array}{c}\bar{v}_{\exp } \\
\text { oder } v_{\exp } \\
\mathrm{MHz}\end{array}$ & $\begin{array}{r}v_{\text {ber }} \\
\mathrm{MHz}\end{array}$ & $\begin{array}{l}\Delta v_{\exp } \\
\mathrm{MHz}\end{array}$ & $\begin{array}{l}\Delta v_{\text {ber }} \\
\mathrm{MHz}\end{array}$ & $\underset{\mathrm{kHz}}{\bar{v}_{\exp }-v_{\text {ber }}}$ & $\begin{array}{l}\text { Bemer- } \\
\text { kungen }\end{array}$ \\
\hline $3_{03}-4_{14}$ & $\begin{array}{l}5-4 \\
4-3\end{array}$ & $\begin{array}{l}17643,71 \\
17643,05\end{array}$ & 17643,52 & 17643,462 & $\begin{array}{r}0,19 \\
-0,47\end{array}$ & $\begin{array}{r}0,18 \\
-0,46\end{array}$ & +58 & ec \\
\hline $3_{12}-4_{23}$ & $\begin{array}{l}5-4 \\
4-3 \\
3-2\end{array}$ & $\begin{array}{l}25984,49 \\
25983,56 \\
25984,83\end{array}$ & 25984,26 & $25,984,266$ & $\begin{array}{r}0,23 \\
-0,70 \\
0,55\end{array}$ & $\begin{array}{r}0,24 \\
-0,71 \\
0,56\end{array}$ & -6 & $\mathrm{ec}, \mathrm{hfs}$ \\
\hline $3_{13}-4_{04}$ & $\begin{array}{l}5-4 \\
4-3 \\
3-2\end{array}$ & $\begin{array}{l}14928,37 \\
14928,56 \\
14928,22\end{array}$ & 14928,40 & 14928,391 & $\begin{array}{r}-0,03 \\
0,16 \\
-0,18\end{array}$ & $\begin{array}{r}-0,03 \\
0,16 \\
-0,19\end{array}$ & + & $\mathrm{ec}, \mathrm{hfs}$ \\
\hline $3_{22}-4_{13}$ & $\begin{array}{l}5-4 \\
4-3 \\
3-2\end{array}$ & $\begin{array}{l}10831,66 \\
10832,40 \\
10831,49\end{array}$ & 10831,86 & 10831,857 & $\begin{array}{r}-0,20 \\
0,54 \\
-0,37\end{array}$ & $\begin{array}{r}-0,19 \\
0,52 \\
-0,37\end{array}$ & +3 & $\mathrm{ec}, \mathrm{hfs}$ \\
\hline $4_{40}-4_{31}$ & & & 24465,00 & 24464,977 & & & +23 & $\mathrm{ec}$ \\
\hline $4_{31}-4_{22}$ & $\begin{array}{l}5-5 \\
4-4 \\
3-3\end{array}$ & $\begin{array}{l}16368,56 \\
16368,06 \\
16368,67\end{array}$ & 16368,42 & 16368,438 & $\begin{array}{r}0,14 \\
-0,36 \\
0,25\end{array}$ & $\begin{array}{r}0,13 \\
-0,35 \\
0,25\end{array}$ & -18 & $\mathrm{ec}, \mathrm{hfs}$ \\
\hline $4_{22}-4_{13}$ & & & 8731,91 & 8731,905 & & & +5 & ec, st \\
\hline $4_{13}-4_{04}$ & $\begin{array}{l}5-5 \\
4-4 \\
3-3\end{array}$ & $\begin{array}{l}9038,22 \\
9039,91 \\
9037,80\end{array}$ & 9038,68 & 9038,649 & $\begin{array}{r}-0,46 \\
1,23 \\
-0,88\end{array}$ & $\begin{array}{r}-0,44 \\
1,22 \\
-0,87\end{array}$ & +31 & $\mathrm{ec}, \mathrm{hfs}$ \\
\hline $4_{32}-4_{23}$ & & & 18124,24 & 18124,282 & & & -42 & ec \\
\hline $4_{23}-4_{14}$ & $\begin{array}{l}5-5 \\
4-4 \\
3-3\end{array}$ & $\begin{array}{l}14887,42 \\
14888,60 \\
14887,11\end{array}$ & 14887,73 & 14887,739 & $\begin{array}{r}-0,31 \\
0,87 \\
-0,62\end{array}$ & $\begin{array}{r}-0,31 \\
0,86 \\
-0,62\end{array}$ & -9 & ec, hfs \\
\hline $5_{32}-5_{23}$ & $\begin{array}{l}6-6 \\
5-5\end{array}$ & $\begin{array}{l}15284,88 \\
15284,40\end{array}$ & 15284,75 & 15284,772 & $\begin{array}{r}0,13 \\
-0,35\end{array}$ & $\begin{array}{r}0,14 \\
-0,37\end{array}$ & -22 & \\
\hline $5_{23}-5_{14}$ & $\begin{array}{l}6-6 \\
5-5\end{array}$ & $\begin{array}{l}9186,77 \\
9187,17\end{array}$ & 9186,89 & 9186,923 & $\begin{array}{r}-0,12 \\
0,28\end{array}$ & $\begin{array}{r}-0,11 \\
0,28\end{array}$ & -33 & st \\
\hline $5_{14}-5_{05}$ & $\begin{array}{l}6-6 \\
5-5\end{array}$ & $\begin{array}{l}12336,98 \\
12338,75\end{array}$ & 12337,48 & 12337,487 & $\begin{array}{r}-0,50 \\
1,27\end{array}$ & $\begin{array}{r}-0,48 \\
1,25\end{array}$ & -7 & \\
\hline $6_{33}-6_{24}$ & & & 14155,84 & 14155,886 & & & -46 & \\
\hline $6_{24}-6_{15}$ & $\begin{array}{l}7-7 \\
6-6\end{array}$ & $\begin{array}{l}10515,70 \\
10516,42\end{array}$ & 10515,91 & 10515,964 & $\begin{array}{r}-0,21 \\
0,51\end{array}$ & $\begin{array}{r}-0,20 \\
0,50\end{array}$ & -54 & \\
\hline $7_{34}-7_{25}$ & & & 13379,00 & 13379,035 & & & -35 & \\
\hline $7_{25}-7_{16}$ & $\begin{array}{l}8-8 \\
7-7\end{array}$ & $\begin{array}{l}19836,75 \\
12837,67\end{array}$ & 12837,01 & 12837,189 & $\begin{array}{r}-0,28 \\
0,66\end{array}$ & $\begin{array}{r}-0,28 \\
0,68\end{array}$ & -179 & \\
\hline $8_{35}-8_{26}$ & & & 13326,09 & 13326,142 & & & -52 & \\
\hline $9_{36}-9_{27}$ & $\begin{array}{c}10-10 \\
9-9\end{array}$ & $\begin{array}{l}14260,16 \\
14260,53\end{array}$ & 14260,27 & 14260,445 & $\begin{array}{r}-0,11 \\
0,26\end{array}$ & $\begin{array}{r}-0,12 \\
0,28\end{array}$ & -175 & \\
\hline
\end{tabular}

Rotationskonstanten (Effektivwerte) und $\tau$ mit Standardfehlern aus (5.3).

$A^{\prime}=(5766,657 \pm 0,003) \mathrm{MHz} ; B^{\prime}=(2632,538 \pm 0,001) \mathrm{MHz} ; C^{\prime}=(1820,751 \pm 0,001) \mathrm{MHz} ; \tau=-(0,0242 \pm 0,0002) \mathrm{MHz}$

Tab. 1. Übergänge $m=0$. Die Frequenzen $\bar{\nu}_{\text {exp }}$ von Linien mit aufgelöster Hyperfeinstruktur wurden durch intensitätsgewichtete Mittelung über die einzelnen HFS-Komponenten berechnet ${ }^{11}$. Bei nur partiell aufgelöster HFS sind unter $v_{\text {exp }}$ nur die intensivsten Komponenten angegeben. Die Frequenzen $\nu_{\text {ber }}$ wurden mit den effektiven Rotationskonstanten $A^{\prime}, B^{\prime}, C^{\prime}$ und der Korrekturkonstanten $\tau$, die aus einer Anpassung der mit ec gekennzeichneten Linien resultieren, nach (5.3) berechnet. Unter $\Delta v_{\text {exp }}$ und $\Delta v_{\text {ber }}$ finden sich die gemessenen und nach (7.2) berechneten Frequenzablagen der einzelnen Multiplettkomponenten von der ungestörten Frequenz $\bar{\nu}_{\text {exp }}$. hfs: Linienaufspaltungen, die zur Bestimmung der Quadrupolkopplungskonstanten verwendet wurden (s. Tab. 4); st: Stark-Effekt der Linien wurde zur Bestimmung der Dipolmomentkomponenten verwendet (s. Tab. 4).

terner und äußerer Rotation modifizierten Rotationskonstanten von dem des Moleküls bei eingefrorener interner Rotation abweicht.

11 H. D. Rudolph, Z. Naturforsch. 23 a, 540 [1968].

$$
m \neq 0 \text {. }
$$

Die Frequenzen der Rotationsübergänge für $m \neq 0$ wurden nach dem Gesamt-Hamilton-Operator (2) berechnet. Eine Zuordnung der $2 J+1$-Subniveaus für festes $J$ zu der durch die Quantenzahlen $K_{-} K_{+}$be- 
stimmten Niveaufolge eines starren asymmetrischen Kreisels ist für $m \neq 0$ nicht mehr möglich ${ }^{13}$, da die entsprechenden Submatrizen stark von der Energiematrix eines starren Kreisels abweichen.

Entsprechend werden auch die Auswahlregeln und Linienstärken des starren asymmetrischen Kreisels modifiziert. Wegen der Vielfalt der auf Grund der Auswahlregeln $\mathrm{E} \longleftrightarrow \mathrm{E}, \quad \mathrm{A}_{1} \longleftrightarrow \mathrm{A}_{2}$ möglichen Übergänge war eine Berechnung der Übergangswahrscheinlichkeiten erforderlich. Für das 2MP lassen sich noch einige speziellere Aussagen über die Niveaubezeichnung und die zu erwartenden Übergangswahrscheinlichkeiten machen. Wegen des kleinen Winkels $\delta$ zwischen interner Rotationsachse und $a$-Achse $\left(\lambda_{b} \ll 1\right)$ - er wurde zu $0,85^{\circ}$ bestimmt lassen sich die Energiezustände angenähert nach der Parität (gerade oder ungerade) der Projektionsquantenzahl $K$ des symmetrischen langgestreckten Kreisels (prolate top) klassifizieren, da dann die Matrixelemente, die Zustände verschiedener Parität von $K$ verbinden (auf Grund des Operators $P_{b}$ ), klein sind. Die Rechnung ergibt ein Verhältnis von ca. 100 : 1 der Anteile von Basisfunktionen verschiedener Parität von $K$, die in einen Zustand eingehen. Damit läßt sich genähert zwischen reinen $a$ - und $b$ Dipolübergängen unterscheiden ${ }^{\mathbf{1 4}}$.

Ferner läßt sich im Falle des 2MP der Betrag von $K$ zur Niveaubezeichnung verwenden, obwohl wegen der Asymmetrie des Moleküls $(x=-0,58)$ Beimischungen anderer $K$ (gleicher Parität) merklich sind. Für die E-Spezies lassen sich die Niveaus dann durch $|K|,|m|$ und das Vorzeichen von $K \cdot m$ $[\operatorname{sgn}(K m)]$ kennzeichnen, weil durch die Entartung $E(-K,-m)=E(K, m)$ die eine Hälfte der zuständigen Matrix von vornherein gestrichen werden kann, wobei die eigentlich mögliche Doppeldeutigkeit $\operatorname{sgn}(+K \cdot(-m))=\operatorname{sgn}(-K \cdot(+m))$ gerade entfällt. Für die zu den Spezies $A_{1}$ und $A_{2}$ von $D_{3}$ gehörigen Eigenwerte ist die Entartung durch das Potential aufgehoben. Für das Niveau $|m|=3$ der reinen internen Rotation resultiert eine Aufspaltung von ca. $530 \mathrm{GHz}$, entsprechend dem Abstand der Torsionsniveaus $v=1$ und $v=2$ (A-Spezies) (vgl. Abb. 2) für den Grenzfall hohen Hinderungspotentials ${ }^{6}$, so daß sich die zugehörigen Rotationssub-

13 Außer für sehr hohe Hinderungspotentiale, wo sich für die einzelnen Torsionsniveaus die Spektren quasi-starrer asymmetrischer Kreisel ergeben.

14 Für $\lambda_{b}=0$ in (2) ergibt sich eine höhere Symmetrie des Hamilton-Operators: $D_{3}$. Dann läßt sich streng zwischen $a$-Dipolübergängen (keine Paritätsänderung von $K$ ) und niveaus für nicht zu hohes $J$ nicht überlappen. Für $|m|=3$ lassen sich also neben $|K|,|m|$ und Spezieskennzeichnung $\delta$ auch die Torsionsquantenzahlen $v=1,2$ zur Niveaukennzeichnung angeben.

Die Rotationsübergänge $|m|=1$ und $|m|=2$ (ESpezies) ließen sich vollständig zuordnen. Zuordnungshilfen waren der lineare Stark-Effekt, die Hyperfeinstruktur der Linien (vgl. folgenden Abschnitt), sowie für das $|m|=1$-Spektrum der aus dem $m=0$ Spektrum entnommene Schätzwert für ein effektives $V_{3}$, der durch die Messungen innerhalb der Fehlergrenzen bestätigt wurde. Die größte Information über das Potential ließ sich aus den intensiven $|\Delta K|=1$-Übergängen („,b-Dipolübergänge“) gewinnen. Es zeigte sich, daß die gemessenen Spektren $|m|=1$ und $|m|=2$ sich nicht allein durch einen $V_{3}$-Term in der Potentialentwicklung wiedergeben lassen. Erst die Berücksichtigung eines $V_{6}$-Termes erlaubt die simultane Wiedergabe der gemessenen $|m|=1$ - und $|m|=2$-Linien auf Grund des verwendeten Modells. Überraschenderweise ließen sich die gemessenen Frequenzen durch zwei verschiedene Sätze für $V_{3}$ und $V_{6}$ wiedergeben, die beide etwa gleich gute Passung ergaben, sich aber sowohl in der Größe der beiden Potentialkoeffizienten als auch im Vorzeichen von $V_{6}$ unterscheiden ${ }^{15}$ :

$$
\begin{array}{ccc}
\text { Satz } & V_{3} \text { in cal/Mol } & V_{6} \text { in cal/Mol } \\
\text { I } & 258,4 \pm 0,1 & -11,8 \pm 0,1 \\
\text { II } & 186,8 \pm 0,5 & 183,3 \pm 0,5
\end{array}
$$

Die Güte der Anpassung, die Úbergangswahrscheinlichkeiten und Hyperfeinstrukturmuster unterscheiden sich für beide Sätze nicht signifikant, so daß sich keiner der beiden möglichen Potentialsätze aus der Anpassung an die $|m|=1$ - und $|m|=2$-Linien bevorzugen läßt. Bezieht man allerdings die Rotationsübergänge für den Grundzustand $m=0$ in die Anpassung für $V_{3}$ und $V_{6}$ mit ein, so ergibt sich für den Satz II eine wesentlich schlechtere Passung der berechneten zu den gemessenen Frequenzen. Während für den Satz I die Standardabweichung klein bleibt $(240 \mathrm{kHz})$, ergibt sich für den Satz II, auch bei gleichzeitiger Anpassung der Rotationskonstanten, eine Standardabweichung von 1,7 MHz. Einzelne Linien für $m=0$ weichen bis zu $3 \mathrm{MHz}$ von

$b$-Dipolübergängen (Paritätsänderung von $K$ ) unterscheiden.

15 Der gleiche Sachverhalt wurde für das meta-Fluortoluol ${ }^{8}$ und das N-Methylpyrrol (vgl. W. ARNold, H. Dreizler u. H. D. Rudolph, Z. Naturforsch. 23 a, 301 [1968]) gefunden. 


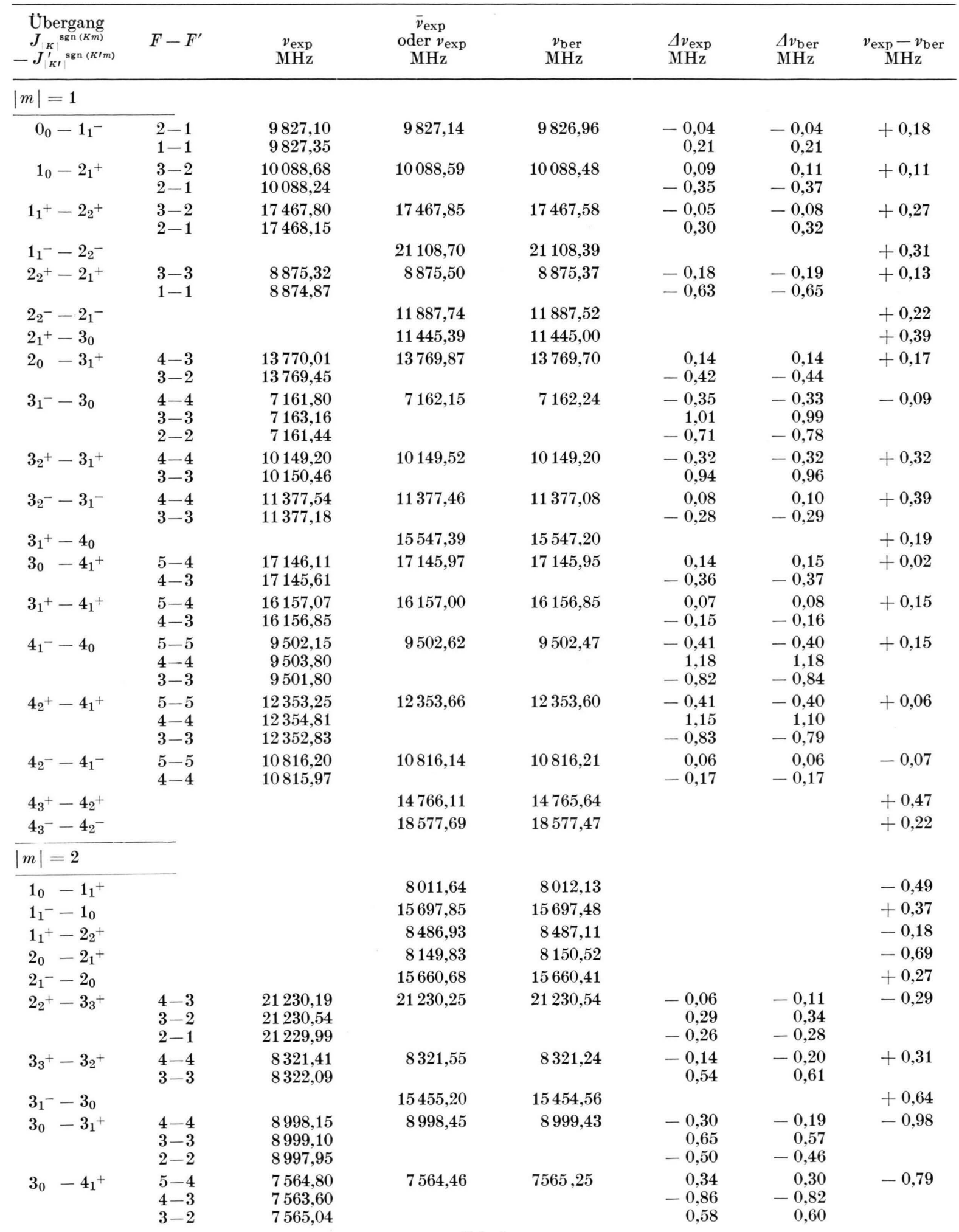

Tab. 2. 


\begin{tabular}{|c|c|c|c|c|c|c|c|}
\hline 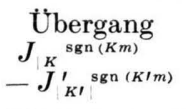 & $F-F^{\prime}$ & $\begin{array}{l}v_{\exp } \\
\mathrm{MHz}\end{array}$ & $\begin{array}{c}\bar{v}_{\exp } \\
\text { oder } v_{\exp } \\
\text { MHz }\end{array}$ & $\begin{array}{l}v_{\text {ber }} \\
\mathrm{MHz}\end{array}$ & $\begin{array}{l}\Delta v_{\exp } \\
\mathrm{MHz}\end{array}$ & $\begin{array}{l}\Delta v_{\text {ber }} \\
\mathrm{MHz}\end{array}$ & $\begin{array}{c}v_{\text {exp }}-v_{\text {ber }} \\
\mathrm{MHz}\end{array}$ \\
\hline $4_{3}^{+}-4_{2}^{+}$ & $\begin{array}{l}5-5 \\
4-4 \\
3-3\end{array}$ & $\begin{array}{l}10258,08 \\
10259,40 \\
10257,80\end{array}$ & 10258,44 & 10258,94 & $\begin{array}{r}-0,36 \\
0,96 \\
-0,64\end{array}$ & $\begin{array}{r}-0,35 \\
0,95 \\
-0,67\end{array}$ & $-0,50$ \\
\hline $\mathbf{4}_{0}-\mathbf{4}_{1}^{+}$ & $\begin{array}{l}5-5 \\
4-4\end{array}$ & $\begin{array}{l}10939,42 \\
10940,69\end{array}$ & 10939,76 & 10941,25 & $\begin{array}{r}-0.34 \\
0,93\end{array}$ & $\begin{array}{r}-0,33 \\
0,92\end{array}$ & $-1,49$ \\
\hline $\begin{array}{l}4_{1}-4_{0} \\
|m|=3\end{array}$ & & & 15062,24 & 15061,97 & & & $+0,27$ \\
\hline \multicolumn{8}{|c|}{$\left(J_{|K|}^{\delta}-J^{\prime}{ }_{K^{\prime}}^{\delta \prime}\right)^{m}{ }_{v}$} \\
\hline$\left(1_{0}^{1}-2_{1}^{1}\right)^{3}{ }_{1}$ & $\begin{array}{l}3-2 \\
2-1\end{array}$ & $\begin{array}{l}9563,70 \\
9562,80\end{array}$ & 9563,50 & $\begin{array}{c}9526,14 \\
(9534,62)\end{array}$ & $\begin{array}{r}0,20 \\
-0,70\end{array}$ & $\begin{array}{r}0,21 \\
-0,71\end{array}$ & $\begin{array}{c}+37,36 \\
(+28,88)\end{array}$ \\
\hline$\left(3_{2}{ }^{0}-3_{1}\right)^{3}{ }_{1}$ & $\begin{array}{l}4-4 \\
3-3 \\
2-2\end{array}$ & $\begin{array}{l}8158,25 \\
8159,42 \\
8157,80\end{array}$ & 8158,53 & $\begin{array}{c}8121,05 \\
(8148,33)\end{array}$ & $\begin{array}{r}0,28 \\
0,89 \\
-0,73\end{array}$ & $\begin{array}{r}-0,29 \\
0,89 \\
-0,71\end{array}$ & $\begin{array}{c}+37,48 \\
(+10,20)\end{array}$ \\
\hline$\left(3_{0}^{0}-4_{1}^{0}\right)^{3}{ }_{1}$ & $\begin{array}{l}5-4 \\
4-3\end{array}$ & $\begin{array}{l}16238,08 \\
16237,73\end{array}$ & 16237,98 & $\begin{array}{c}16232,83 \\
(16237,73)\end{array}$ & $\begin{array}{r}0,10 \\
-0,25\end{array}$ & $\begin{array}{r}0,13 \\
-0,29\end{array}$ & $\begin{array}{c}+5,15 \\
(+0,25)\end{array}$ \\
\hline
\end{tabular}

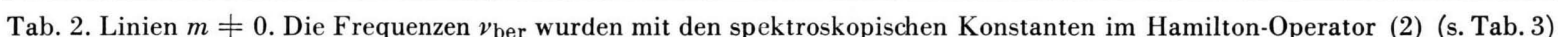
berechnet, die aus einer Anpassung der Linien $|m|=0,1,2$ resultieren. Die in Klammern gesetzten Frequenzwerte bei $|m|=3$ ergaben sich bei Einbeziehung dieser Linien in die Anpassung für $V_{3}$ und $V_{6}\left(V_{3}=258,3 \mathrm{cal} / \mathrm{Mol}, V_{6}=-12,5 \mathrm{cal} / \mathrm{Mol}\right)$. Die HFS-Aufspaltungen wurden nach (7.2) mit den aus dem $m=0$-Spektrum entnommenen Kopplungskonstanten (Tab. 4) berechnet.
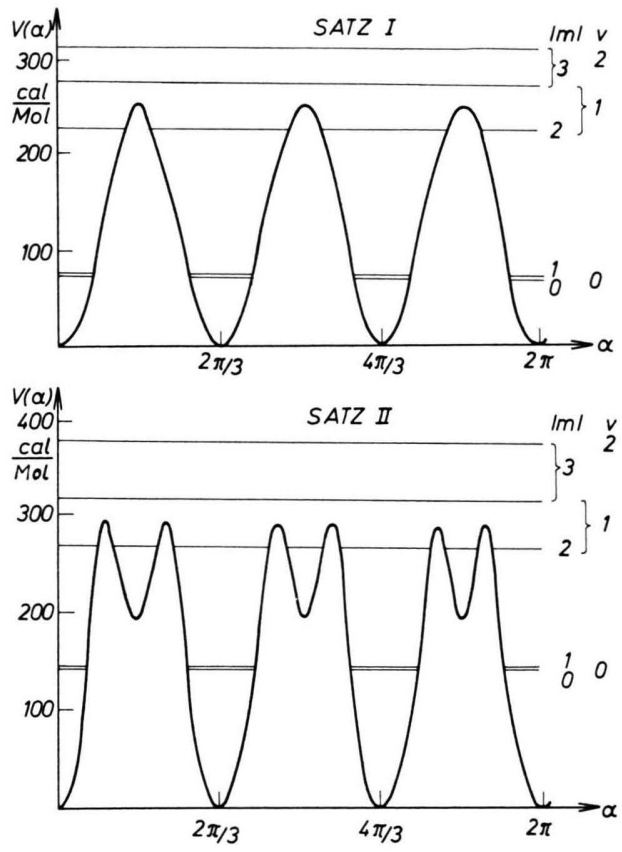

Abb. 2. Mögliche Potentialformen $V(\alpha)=\frac{1}{2} V_{3}(1-\cos 3 \alpha)$ $+\frac{1}{2} V_{6}(1-\cos 6 \alpha)$ des 2 MP aus der Anpassung an die gemessenen $|m|=1$ - und $|m|=2$-Linien mit den Energieniveaus der reinen internen Rotation $(J=0)$. [ $[|m|$ : Quantenzahl des freien Rotators, $v$ : Quantenzahl des harmonischen Oszillators, $V(a) \rightarrow \infty$.]

den berechneten Frequenzen ab. Dies ließe sich nur durch sehr grobe Mängel des Modells erklären. Da sich aber für alle bisherigen Moleküle mit interner Rotation zumindest das Spektrum für $m=0$ gut nach dem verwendeten Modell wiedergeben läßt, wurde der Satz II für die Potentialkoeffizienten $V_{3}$ und $V_{6}$ im weiteren ausgeschlossen.

Neben $V_{3}$ und $V_{6}$ wurden aus den $|m|=0,1,2$ Spektren die Rotationskonstanten $A, B, C$ des Gesamtmoleküls, der Winkel $\delta$ zwischen interner Rotationsachse und $a$-Achse und das Trägheitsmoment $I_{\alpha}$ der Methylgruppe bestimmt.

$$
\begin{aligned}
& A=(5695,77+0,06) \mathrm{MHz} \quad \delta=(0,85 \pm 0,01)^{0} \\
& B=(2632,55 \pm 0,08) \mathrm{MHz} \quad I_{\alpha}=(3,19 \pm 0,01) \mathrm{AME} \cdot \AA^{2} \\
& C=(1820,65 \pm 0,04) \mathrm{MHz} \\
& V_{3}=(2710 \pm 1) \mathrm{GHz}=(258,4 \pm 0,1) \mathrm{cal} / \mathrm{Mol} \\
& V_{6}=-(123 \pm 1) \mathrm{GHz}=-(11,8 \pm 0,1) \mathrm{cal} / \mathrm{Mol}
\end{aligned}
$$

Tab. 3. Spektroskopische Konstanten des 2 MP mit Standardfehlern.

Höhere Potentialkoeffizienten als $V_{3}$ und $V_{6}$ ließen sich aus den Spektren $|m|=1$ und $|m|=2$ nicht bestimmen, da sie völlig mit den beiden ersten Potentialtermen korrelierten. Das Rotationsspektrum für $|m|=3$ ließ sich nicht vollständig zuordnen. Es ließen sich nur die drei angegebenen Linien finden, die im Stark-Effekt und HFS-Muster mit der Rechnung übereinstimmen und in dem zu erwartenden Frequenzgebiet liegen. Bezieht man diese Linien in die Anpassung für $V_{3}$ und $V_{6}$ mit ein, so ergibt sich für 
$V_{3}=(258,3 \pm 0,1) \mathrm{cal} / \mathrm{Mol}$ ein Wert, der innerhalb der Fehlergrenzen des nur aus dem $|m|=1$ - und $|m|=2$-Spektrum ermittelten Wert liegt. $V_{6}$ ändert sich jedoch mit $(-12,5 \pm 0,2) \mathrm{cal} / \mathrm{Mol}$ um $0,7 \mathrm{cal}$ /Mol. Der Standardfehler wird mit $6,5 \mathrm{MHz}$ rund 15-mal größer als bei der Anpassung von $V_{3}$ und $V_{6}$ an die $|m|=1$ - und $|m|=2$-Spektren allein und ließ sich auch nicht durch Einbeziehung höherer Potentialterme verringern, da diese fast völlig mit $V_{\mathbf{3}}$ und $V_{6}$ korreliert sind. Die große Standardabweichung von $6,5 \mathrm{MHz}$ ist also nicht in der Vernachlässigung höherer Potentialkoeffizienten zu suchen, da sie sich bei deren Anpassung hätte verringern müssen, sondern ist vielmehr den Mängeln des Modells zuzuschreiben, in dem keine Wechselwirkung von interner Rotation und Molekülschwingung berücksichtigt wird. Die Raman- und IR-Spektren ${ }^{16}$ des 2MP zeigen beide eine Absorptionslinie bei ca. $200 \mathrm{~cm}^{-1}$ ( $\triangleq 570 \mathrm{cal} / \mathrm{Mol}$ ), die vermutlich der Knickschwingung Methylgruppe-Ring zuzuschreiben ist. Der Einfluß dieser Schwingung auf die Rotations-Torsionsniveaus läßt sich nicht exakt abschätzen. Die gute Passung der $|m|=0,1,2$-Linien an das verwendete Molekülmodell und die nur partielle Zuordnung der $|m|=3$-Linien und deren schlechte Passung legen jedoch die Vermutung nahe, daß die höher angeregten internen Rotationszustände stärker von einer eventuellen Wechselwirkung von interner Rotation und Schwingung beeinflußt sind. Aus diesen Gründen haben wir die $|m|=3$-Linien bei der Anpassung für $V_{3}$ und $V_{6}$ ausgenommen. Nach höher angeregten internen Rotationszuständen als $|m|=3$ wurde nicht gesucht, da der BoltzmannFaktor dann bereits erheblich zum Tragen kommt und des weiteren die gemachten Einschränkungen über die Güte des verwendeten Modells zu beachten sind.

\section{Kernquadrupolkopplungskonstanten und Dipolmoment}

Die Linien zeigen eine ausgeprägte Multiplettstruktur auf Grund der Wechselwirkung von Molekülrotation und Kernspin des Stickstoffkernes $\left({ }^{14} \mathrm{~N}\right)$ über das Kernquadrupolmoment und den Feldgra-

\footnotetext{
16 Das Raman-Spektrum wurde am Chemischen Institut der Universität Bologna von Herrn Dr. A. Guarnieri, das IRSpektrum am Phys.-Chem. Institut der Universität Freiburg von Frl. Dr. B. STARCK aufgenommen.

17 J. K. Bragg, Phys. Rev. 74, 533 [1948].

18 J. K. Bragg u. S. Golden, Phys. Rev. 75, 735 [1949].
}

dienten der Elektronenhülle am Kernort. Die Störenergie auf Grund eines quadrupolmomentbehafteten Kernes ist in 1. Ordnung gegeben durch ${ }^{17,18}$

$$
E_{Q}=\frac{2 J+3}{J} f(I, J, F) \sum_{g, g^{\prime}}\left\langle\lambda_{Z g} \lambda_{Z g^{\prime}}\right\rangle_{J \xi M_{J}=J} \chi_{g g^{\prime}}
$$

$\lambda_{\mathrm{Zg}}$ Richtungskosinus zwischen raumfester $Z$-Achse und molekülfester $g$-Achse,

$\chi_{g g^{\prime}}$ Komponenten des Quadrupolkopplungstensors bezüglich der $g$-Hauptachsen des Moleküls,

$\xi \quad$ Numerierungsindex zur Kennzeichnung der Rotationsniveaus für festes $J$ (entsprechend $K_{-} K_{+}$ beim starren asymmetrischen Kreisel),

\section{$f(I, J, F) \quad$ Casimir-Funktion.}

Für den starren asymmetrischen Kreisel verschwinden die Diagonalelemente der Mischglieder $\left(\lambda_{Z g} \lambda_{Z g^{\prime}}\right)$ $\left(g \neq g^{\prime}\right)$ aus Symmetriegründen und man erhält für die Zusatzenergie eines Rotationszustandes $\left(J K_{-} K_{+}\right)$ auf Grund der Quadrupolwechselwirkung den bekannten Ausdruck:

$$
E_{Q}=\frac{2}{J(J+1)} f(I, J, F) \sum_{g=a, b, c} \chi_{g g}\left\langle P_{g}^{2}\right\rangle_{J, \xi} .
$$

Wir sind bei der Bestimmung der $\chi_{g g}$ nach (7.2) aus den $m=0$-Linien von den Eigenfunktionen des starren asymmetrischen Kreisels $\left(\xi=K_{-} K_{+}\right)$ausgegangen, da eine Berücksichtigung des zu $P_{a}{ }^{4}$ proportionalen Korrekturtermes in (5.2) keinen meßbaren Einfluß ergibt. Die genaue Auswertungsmethode ist an anderer Stelle ausführlich dargestellt ${ }^{19}$. Die Ergebnisse finden sich in Tab. 4.

Für angeregte interne Rotationszustände verschwinden die Diagonalelemente der Mischglieder $\lambda_{Z a} \cdot \lambda_{Z b}$ im Eigenfunktionssystem des Hamilton-Operators (2) nicht ${ }^{20}$, und der Ausdruck (7.2) für die Quadrupolzusatzenergie muß durch einen additiven Term - $+[(4 J+6) / J]\left\langle\lambda_{Z a} \lambda_{Z b}\right\rangle_{J, \xi}-$ ergänzt werden.

Für den speziellen Fall des 2MP erweist sich dieser Beitrag jedoch als vernachlässigbar klein und zur Berechnung der Hyperfeinstruktur findet Formel (7.2) Anwendung, wobei allerdings die Erwartungswerte der $P_{g}{ }^{2}$ in den einzelnen Rotations-Torsionszuständen $(J, \xi)$ zu berechnen sind. Sie sind gegen-

19 H. D. Rudolph, H. Dreizler u. H. Seiler, Z. Naturforsch. 22 a, 1738 [1967].

$20 \lambda_{Z a} \lambda_{Z b}$ gehört der totalsymmetrischen Spezies von $\mathbf{A}_{1}$ von $\mathrm{D}_{3}$ an. Für $\lambda_{b}=0$ verschwindet der Zusatzterm dann exakt, da Invarianzgruppe $D_{3 h}$. 
über den Erwartungswerten für $m=0$ modifiziert. Da die Quadrupolkopplungskonstanten sich in 1. Näherung für angeregte interne Rotationszustände nicht ändern ${ }^{21}$, ist die Hyperfeinstruktur mit Kenntnis der $\chi_{g g}$ (aus $m=0$ ) für $m \neq 0$ im voraus berechenbar und kann als Zuordnungshilfe verwendet werden. Die Passung der berechneten zu den gemessenen Frequenzaufspaltungen wird allerdings schlechter als für $m=0$, was vermutlich seinen Grund in den Vereinfachungen des zugrunde liegenden Modells hat.

$$
\begin{gathered}
\chi_{a a}=-(0,33 \pm 0,02) \mathrm{MHz}, \quad \chi_{b b}=-(2,86 \pm 0,02) \mathrm{MHz} \\
\chi_{c c}=+(3,19 \pm 0,02) \mathrm{MHz} \\
\left|\mu_{a}\right|=(0,72 \pm 0,01) \mathrm{D}, \quad\left|\mu_{b}\right|=(1,71 \pm 0,02) \mathrm{D} .
\end{gathered}
$$

Tab. 4. Quadrupolkopplungskonstanten und Dipolmomentkomponenten mit Standardfehlern aus Linien $m=0$.

Neben den Quadrupolkopplungskonstanten wurden dem $m=0$-Spektrum die Information über die
Dipolmomentkomponenten entnommen. Sie wurden nach dem Schema des starren Kreisels an die Frequenzablagen der Stark-Satelliten von drei $m=0$ Linien bei verschiedenen Feldstärken angepaßt, und zwar:

$$
\begin{array}{ll}
3_{2,1}-3_{1,2} & (M=3) ; \\
4_{2,2}-4_{1,3} & (M=4) ; \\
5_{2,3}-5_{1,4} & (M=5) .
\end{array}
$$

Es wurde bei möglichst hohen Feldstärken gemessen, da sich dann der Einfluß der Quadrupolwechselwirkung vernachlässigen läßt.

Wir danken Herrn Dr. G. E. Herberich und Herrn A. Trinkaus für die freundliche Überlassung von Programmen, Frl. Dr. B. Starck und Herrn Dr. A. GuarNIERI für die Aufnahme der IR- und Raman-Spektren des 2 MP. Die numerischen Rechnungen wurden am Rechenzentrum der Universität Freiburg durchgeführt.

21 R. Lett u. H. Flygare, J. Chem. Phys. 47, 4730 [1967].

\title{
The Rotational Spectra and Dipole Moments of AgF and CuF by Microwave Absorption
}

\author{
J. Hoeft, F. J. Lovas, E. Tiemann, T. TöRring
}

II. Physikalisches Institut der Freien Universität Berlin, Berlin 33

(Z. Naturforsch. 25 a, 35-39 [1970] ; received 23 October 1969)

\begin{abstract}
The rotational transitions $J=1 \rightarrow 2$ and $2 \rightarrow 3$ were measured for ${ }^{107} \mathrm{Ag}^{19} \mathrm{~F}$ and ${ }^{109} \mathrm{Ag}^{19} \mathrm{~F}$ by microwave absorption. These spectra allowed the determination of the Dunham-coefficients $Y_{01}$, $Y_{11}, Y_{21}$, and $Y_{02}$ and from these the potential coefficients $a_{0}$ and $a_{1}$ and internuclear distance $r_{\mathrm{e}}$ were derived. For ${ }^{63} \mathrm{Cu}^{19} \mathrm{~F}$ and ${ }^{65} \mathrm{Cu}^{19} \mathrm{~F}$ the hyperfine structure was observed in the $J=0 \rightarrow 1$ and $1 \rightarrow 2$ rotational spectra. In addition to the Dunham coefficients the quadrupole coupling constants, e $q Q$, and spin-rotation coupling constant, $c$, for the Cu nucleus were obtained. Stark effect measurements on both $\mathrm{AgF}$ and $\mathrm{CuF}$ resulted in the determination of the electric dipole moment for the ground vibrational state.
\end{abstract}

Since the advent of high temperature microwave spectroscopy, the alkali halides, or the group $\mathrm{I}_{\mathrm{a}}$-VII diatomic molecules, have been studied in great detail. However, the first MW rotational study of a member of the $I_{b}$-VII sub-group appeared only recently. That study of $\mathrm{AgCl}^{1}$ was followed quickly with a similar investigation of $\mathrm{AgBr}^{2}$, but no new studies have since appeared. Rotational investigations and especially Stark effect measurements on this sub-family of the alkali halides are highly desirable from a theoretical view point; however, this

Sonderdruckanforderungen an Dr. J. HoEfT, II. Physikalisches Institut der FUB, D-1000 Berlin 33, Boltzmannstr. 20.

1 L. C. Krisher and W. G. Norris, J. Chem. Phys. 44, 391 [1966]. - E. PEARSON and W. GordY, Phys. Rev. 152, 42 [1966]. group presents experimental difficulties since many of these monohalides are thermally unstable or chemically reactive when in contact with hot surfaces. The silver and copper monofluorides appear more reactive and unstable than their other monohalides and have required some special techniques for spectral observation.

Our initial observations were made in the molecular beam spectrometer which was employed in earlier measurements ${ }^{3}$. About 5 grams of $\mathrm{AgF}$ (80\% pure material) were place in the tantalum oven. The

2 L. C. Krisher and W. G. Norris, J. Chem. Phys. 44, 974 [1966].

3 T. TörRING, Z. Naturforsch. 23 a, 777 [1968]. 